\title{
ArTícUlo
}

\section{Morfología del sistema mecanosensorial de la línea lateral de Zearaja chilensis (Batoidea: Rajidae)}

\author{
Morphology of the mechanosensory system of the lateral line system in \\ Zearaja chilensis (Batoidea: Rajidae) \\ Sylvia Sáez ${ }^{1}$, Julio Lamilla ${ }^{1}$ y Germán Pequeño ${ }^{1}$ \\ ${ }^{1}$ Instituto de Ciencias Marinas y Limnológicas, Universidad Austral de Chile, Casilla 567, Valdivia, Chile. sylvia.saez@gmail.com
}

\begin{abstract}
The mechanosensory system of the lateral line in Zearaja chilensis, is composed by a series of sensory canals located at both the ventral and dorsal surfaces of the body, being in the latter where the canals are more abundant. The hyomandibular canal is the most complex due to its distribution and extension, showing changes in its morphology at the dorsal and ventral body sides. The present study of the mechanosensory system of $Z$. chilensis, will help to increase the morphological knowledge about the sensory system of this otherwise scarcely studied species, among Chilean batoids, allowing further taxonomic and systematic comparative studies and providing ways to new behavioral and physiological studies including other cartilaginous fishes not only from Batoidea, but also from Squalomorphii and Holocephalii taxa.
\end{abstract}

Key words: Sensory canals, nervous system, lateral line, Rajidae, Chile

Resumen.- El sistema mecanosensorial de la línea lateral de Zearaja chilensis, está compuesto por una series de canales sensoriales de extensión variable según la posición de cada canal y distribuidos en los lados ventral y dorsal del cuerpo, siendo en este último donde se concentra el mayor número de canales. El canal hiomandibular es el más complejo por su distribución y extensión, cambiando su morfología del lado dorsal del cuerpo con respecto del lado ventral. El presente estudio pretende avanzar en el conocimiento general sobre el sistema sensorial de esta especie, muy poco estudiado en los batoídeos chilenos, permitiendo continuar con estudios comparativos taxonómicos y sistemáticos, así como también aplicarlo en nuevos estudios conductuales y fisiológicos, entre otros, que incluyan otros taxones de peces cartilaginosos, tanto de Batoidea como de Squalomorphii y Holocephalii de Chile.

Palabras clave: Canales sensoriales, sistema nervioso, línea lateral, Rajidae, Chile

\section{INTRODUCCIÓN}

El sistema sensorial en condrictios está conformado por dos sistemas: sistema mecanosensorial de la línea lateral y el sistema electrosensorial (Bleckmann \& Hofmann 1999, Montgomery \& Bodznick 1999, Jordan 2008, Jordan et al. 2009). El sistema mecanosensorial detecta movimientos de agua cercanos a la superficie de la piel, mediante mecanoreceptores que están constituidos por células ciliadas sensoriales cubiertas por una cúpula gelatinosa que se conecta hacia el exterior mediante un sofisticado sistema de canales continuos subepidérmicos (Garman 1888, Roberts 1978, Bleckmann \& Hofmann 1999, Maruska 2001, Jordan et al. 2009, Shibuya \& Tanaka 2012). El sistema electrosensorial, detecta campos eléctricos animados e inanimados mediante eletroreceptores, las ámpulas de Lorenzini, que se encuentran en túbulos subcutáneos conectados hacia el exterior por un canal que aparece hacia la superficie epidérmica como un poro somático
(Raschi 1986, Bleckmann \& Hofmann 1999, Montgomery \& Bodznick 1999, Tricas 2001, Wueringer \& Tibbetts 2008, McGowan \& Kajiura 2009, Jordan et al. 2009, Marzullo et al. 2011, Wueringer et al. 2011). El presente estudio sólo se referirá al sistema mecanosensorial de la línea lateral.

En batoideos, los canales de la línea lateral forman una red subcutánea y pueden ser: porados, los cuales detectan la aceleración del flujo externo hidrodinámico cercano a la piel, o no porados, los cuales son usados en la superficie ventral para detectar la velocidad hidrodinámica y localización de la presa por la función mecanotáctil (Maruska 2001, Maruska \& Tricas 2004, Shibuya et al. 2010, Marzullo et al. 2011). De esta manera, al distribuirse sobre la superficie dorsal y ventral del cuerpo, entregan información imprescindible para los batoídeos que habitan sobre distintos tipos de sustratos (Maruska \& Tricas 1998, Bleckmann \& Hofmann 1999, 
Maruska 2001, Jordan et al. 2009, Shibuya et al. 2010, Shibuya \& Tanaka 2012).

Morfológicamente, el sistema mecanosensorial de la línea lateral varía en complejidad anatómica, en la ramificación y extensión de los canales en los distintos taxones (en especial órdenes) que constituyen el Superorden Batoidea, entregando información taxonómica, biológica, fisiológica, etológica, ecológica y sistemática (Maruska \& Tricas 1998, Bleckmann \& Hofmann 1999, Maruska 2001, Jordan 2008, Jordan et al. 2009, Shibuya et al. 2010, Carvalho \& Ragno 2011).

En los taxones de batoídeos chilenos, el estudio de los canales mecanosensoriales no ha recibido la atención debida. Consecuentemente, el presente trabajo tiene por objetivos sentar las bases para los estudios morfológicos de los canales mecanosensoriales de la línea lateral de rayas en Chile, empezando con Zearaja chilensis (Guichenot, 1848) y describir la forma y extensión de las distintas ramas, con su respectiva nomenclatura, de modo de facilitar la mejor comprensión biológica de Zearaja chilensis y de las posibles comparaciones futuras con otras especies. Para tal efecto, se consideró a Zearaja chilensis por ser una de las especies de raya sobre la cual hay comparativamente más antecedentes y es comercialmente importante. Zearaja chilensis, es una especie morfológicamente muy adaptada para la vida bentónica en fondos blandos (Koen-Alonso et al. 2001). Entre los batoídeos, alcanza tamaños entre 316 y 1680 mm (Lloris \& Rucabado 1991, Bustamante et al. 2012, Reyes \& Hüne 2012). Aunque se sabe que sus aletas pectorales le permiten una natación no distante del fondo, lo habitual es que la mayor parte del tiempo yace sobre el fondo, incluso a veces ligeramente enterrada en el material blando del mismo (i.e., arena) (Lloris \& Rucabado 1991, Reyes \& Hüne 2012). Todo lo anterior sugiere que Zearaja chilensis debe tener buenos mecanismos de información, que le permitan detectar perturbaciones del agua o movimientos por encima de su cuerpo. Por estas características, se postula que $Z$. chilensis debería presentar un mayor desarrollo de canales mecanosensoriales dorsales. De esta manera, el estudio morfológico mecanosensorial de la línea lateral de Z . chilensis, permitirá un mejor análisis de estas estructuras en los batoideos chilenos, incentivando nuevos estudios taxo-sistemáticos para comprender aspectos conductuales y fisiológicos en batoideos, con posibilidades de ser extensibles al resto de los taxones de Squalomorphii y Holocephalii.

\section{Materiales y MÉTODOS}

Se examinaron 6 ejemplares juveniles (3 machos y 3 hembras) de Zearaja chilensis cuya longitud fue entre 70 y 70,5 cm en machos y $70 \mathrm{~cm}$ en hembras. Los ejemplares fueron capturados en el litoral de la zona de pesquería de la Provincia de Valdivia, comprendida desde el límite norte (desembocadura del Río Queule 39²4's, 73¹3’W) hasta el paralelo $41^{\circ} 28,6^{\prime} \mathrm{S}$, dentro de las 5 millas reservadas a la pesca artesanal, con autorización para operar por Resolución Exenta №318 de la Subsecretaria de Pesca entre las fechas 28 de septiembre y 31 de diciembre 2011 (Lamilla \& Flores 2011) ${ }^{1}$.

Para el estudio de los canales mecanosensoriales y con la finalidad de determinar la topografía de tales estructuras, se procedió a una observación macroscópica en fresco mediante contraste de luz y lupa estereoscópica. Adicionalmente, para esquematizar el trayecto de los canales mecanosensoriales, se efectuó una disección subcutánea retirando la piel en ambos lados del cuerpo.

Para la terminología de los canales se siguió a Maruska \& Tricas (2004).

\section{Resultados}

En los ejemplares Zearaja chilensis analizados se observaron 6 canales en el lado dorsal y 4 en el lado ventral del cuerpo. No se observaron diferencias morfológicas ni topográficas de los canales sensoriales entre machos y hembras. Todos los canales observados, estaban formados por un epitelio continuo.

\section{LADO DORSAL (FIG. 1)}

En la superficie dorsal se observó la mayor cantidad de canales (6).

a. Canal supraorbital (CSO): se origina desde la región post orbital, luego se desvía ántero medialmente hacia los ojos y se extiende hacia el rostrum.

b. Canal infraorbital (CIO): ubicado ántero lateralmente entre los espiráculos y los ojos, en la unión del CSO posterior, girando medialmente a nivel de la mitad del ojo.

c. Canal hiomandibular (CHI): canal complejo y extenso especialmente en la superficie dorsal. Se extiende lateralmente desde el CIO a lo largo del margen del disco y continúa posteriormente más allá del nivel de la cintura escapular.

Lamilla J \& H Flores. 2011. Prospección de los recursos Zearaja chilensis, Dipturus trachyderma y Bathyraja albomaculata y el marcaje como una herramienta de estudio de la distribución espacial en el litoral de la Región de Los Ríos. Informe Técnico P. INV. R. EX. N² 2528. Subsecretaría de Pesca, 94 pp. 


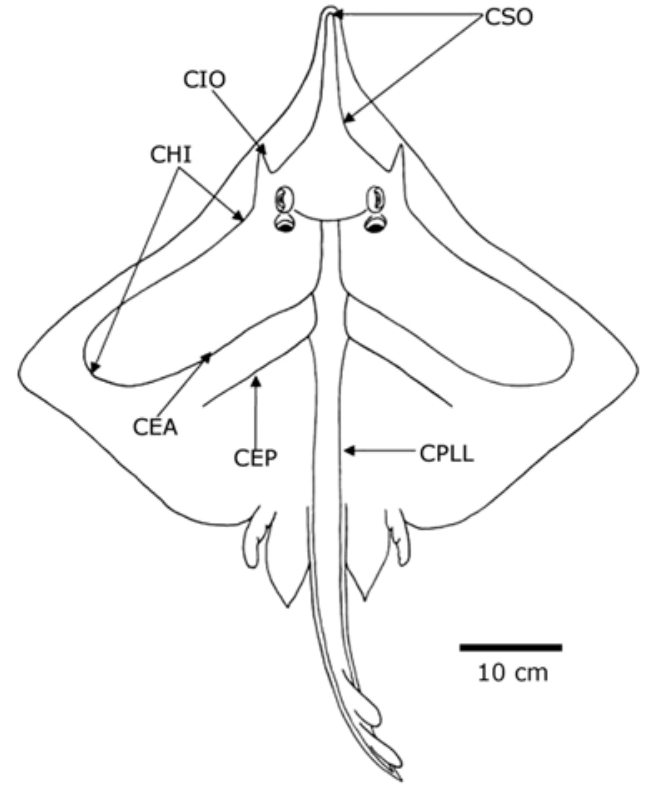

Figura 1. Lado dorsal. Esquema de los canales sensoriales en Zearaja chilensis: CSO canal supraorbital, $\mathrm{ClO}$ canal infraorbital, $\mathrm{CHI}$ canal hiomandibular, CEA canal escapular anterior, CEP canal escapular posterior, CPLL canal posterior de la línea lateral / Dorsal surface. Sensory canals scheme in Zearaja chilensis: CSO supraorbital canal, CIO infraorbital canal, CHI hyomandibular canal, CEA anterior scapular canal, CEP posterior scapular canal, CPLL lateral line posterior canal

d. Canal escapular anterior (CEA): se origina a nivel de la cintura escapular y se curva al extenderse póstero ventralmente más allá de la región central del disco.

e. Canal escapular posterior (CEP): las ramas de este canal, se extienden oblicuamente desde el canal de la línea lateral, alcanzando la región posterior del disco.

f. Canal posterior de la línea lateral (CPLL): canal par, situado por debajo de la cintura escapular hasta la mitad de la aleta pélvica, se extiende caudalmente en línea más o menos recta.

\section{LADO VENTRAL (FIG. 2)}

La superficie ventral del cuerpo presenta 4 canales: prenasal, nasal, mandibular e hiomandibular. Los canales de la superficie ventral están interconectados (excepto el mandibular) e independientemente penetran al disco para unir los canales sobre la superficie ventral. En ejemplares

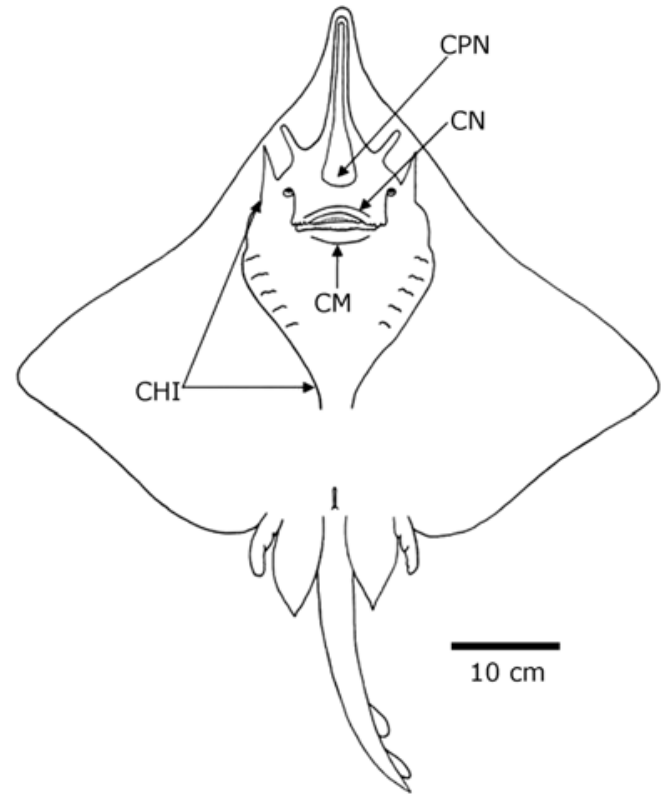

Figura 2. Lado ventral. Esquema de los canales sensoriales en Zearaja chilensis: CPN canal prenasal, $\mathrm{CN}$ canal nasal, CM canal mandibular, $\mathrm{CHI}$ canal hiomandibular / Ventral surface. Sensory canals scheme in Zearaja chilensis: CPN prenasal canal, CN nasal canal, CM mandibular canal, $\mathrm{CHI}$ hyomandibular canal

frescos, los canales CSO y CIO, se ven claramente por transparencia en la superficie ventral.

a. Canal prenasal (CPN): ancho, situado en la fosa prenasal del neurocráneo. Este canal hace un giro entre las narinas al margen del hocico.

b. Canal nasal (CN): corto y ancho, se extiende lateralmente desde la vuelta posterior del canal prenasal a los márgenes externos de la cortina nasal.

c. Canal mandibular (CM): canal ubicado por debajo de la mandíbula inferior.

d. Canal hiomandibular (CHI): localizado a lo largo del margen anterior del disco, continuando medialmente donde se estrecha también medialmente; rodea a las hendiduras branquiales y termina hacia el borde caudal del disco. 


\section{Discusión}

El estudio de los canales mecanosensoriales de la línea lateral en individuos juveniles de Zearaja chilensis, indica que se trata de un sistema conformado por una serie de canales interconectados entre sí, distribuidos sobre la región cefálica y disco, los cuales presentan diferencias en la superficie dorsal y ventral del cuerpo. Los canales observados en el lado dorsal (canal supraorbital, canal infraorbital, canal escapular anterior, canal escapular posterior, hiomandibular y canal posterior de la línea lateral) como en el lado ventral (canal prenasal, nasal, mandibular e hiomandibular) presentaron características similares en forma y distribución a los canales mecanosensoriales registrados en otras especies de Rajiformes y Rajidae, como por ejemplo: Raja chinensis Basilewsky, 1855, Raja laevis Mitchill, 1818, Rioraja agasizzi (Müller \& Henle, 1841), Cruriraja rugosa Bigelow \& Schroeder, 1958, Raja nasuta Müller \& Henle, 1814 (= Zearaja nasuta (Müller \& Henle, 1814) y R. eglanteria Bosc, 1800 (Garman 1888, Roberts 1978, Maruska 2001, Montgomery \& Bodznick 1999, Carvalho et al. 2006).

Entre los canales más extensos en los juveniles de $Z$. chilensis, se encuentra el canal hiomandibular, principalmente en el lado dorsal. Al respecto, se ha descrito que la extensión de este canal, está correlacionada con el largo de las aletas pectorales y del canal supraorbital en un rostro alargado (Maruska \& Tricas 1998, Maruska 2001). Por otro lado, en el lado ventral, el canal hiomandibular de $Z$. chilensis es más estrecho, más cercano a las hendiduras branquiales. Esta estrechez del canal hiomandibular podría estar relacionada con la acción respiratoria, ya que al estar rodeando las hendiduras branquiales, este canal aumenta el contacto con el medio externo y el individuo obtiene mayor información de onda de movimiento del agua. Esta acción es realizada conjuntamente con los otros canales presentes en el lado ventral como canal prenasal, nasal y mandibular, formando un sistema de canales relacionados con la detección de movimientos de agua generados por la locomoción, respiración y actividades de la presa, ayudando a reposicionar el cuerpo hacia la boca directamente sobre la presa (Maruska \& Tricas 1998, Maruska 2001, Marzullo et al. 2011, Shibuya \& Tanaka 2012).

Por su parte, los canales mecanosensoriales dorsales, canal supra e infraorbital y de la línea lateral posterior del cuerpo y cola (canal posterior de la línea lateral, canal escapular anterior y posterior) de Z . chilensis son canales rectos, más cortos en relación al hiomandibular, que bordean el rostrum, ojos y línea lateral. Estas características de los canales mecanosensoriales dorsales, apoyan la hipótesis planteada, confirmando que dichas estructuras sensoriales en $Z$. chilensis permiten detectar perturbaciones del agua o movimientos por encima de su cuerpo. Tal afirmación es apoyada por otros estudios donde se ha demostrado que este complejo sistema de canales dorsales, sirve en la detección de movimientos del agua generados por conespecíficos, obteniendo información sensorial acerca de la conducta del cardumen y poder localizar objetos en el medio ambiente provocados por distorsiones del animal de su propio campo de flujo mientras nada manteniendo la posición del cardumen, aumentando así la sensibilidad sobre la superficie dorsal (Maruska \& Tricas 1998, Maruska 2001, Maruska \& Tricas 2004, Shibuya et al. 2010, Marzullo et al. 2011).

El presente estudio de los canales mecanosensoriales de la línea lateral de Zearaja chilensis, constituye la primera descripción morfológica para esta especie en Chile, y establece un precedente aplicable para el resto de los peces condrictios chilenos. Tal conocimiento ayudará en futuras investigaciones morfológicas que pueden complementarse con aspectos conductuales en diferentes taxones y obtener información comparativa de este importante sistema sensorial, débilmente considerado en batoideos chilenos e integrar esa información en estudios taxonómicos y sistemáticos, no sólo de Batoidea, sino también extensible a los taxones de Squalomorphii y Holocephalii. Naturalmente esta perspectiva también promueve un mejor discernimiento sistemático filogenético de los taxones involucrados.

\section{Agradecimientos}

Los autores agradecen especialmente a Hernán Flores (Biólogo Marino, Laboratorio de Ictiología ELASMOLAB, Instituto de Ciencias Marinas y Limnológicas. Universidad Austral de Chile) por la ayuda prestada durante la elaboración del trabajo y al apoyo de la Dirección de Investigación y Desarrollo de la Universidad Austral de Chile. A Richard L. Haedrich (Memorial University, St. Johns, Canada) y Domingo Lloris (Instituto de Ciencias del Mar, Barcelona, España) proveyeron valioso material bibliográfico. Al dibujante Marcos Navarro por la realización de los esquemas. Estos son resultados parciales del proyecto FIP 2013-29 inscrito en la Dirección de Investigación de la Universidad Austral de Chile. 


\section{LITERATURA CITADA}

Bleckmann H \& MH Hofmann. 1999. Special senses. In: Hamlett WC (ed). Sharks, skates and rays. The biology of elasmobranch fish, pp. 300-327. Johns Hopkins Press, Baltimore.

Bustamante C, C Vargas-Caro, MC Oddone, F Concha, H Flores, J Lamilla \& MB Bennett. 2012. Reproductive biology of Zearaja chilensis (Chondrichthyes: Rajidae) in the south-east Pacific Ocean. Journal of Fish Biology 80(5): 1213-1226.

Carvalho MR \& M Ragno. 2011. An unusual, dwarf new species of Neotropical freshwater stingray, Plesiotrygon nana sp. nov., from the upper and mid Amazon basin: the second species of Plesiotrygon (Chondrichthyes: Potamotrygonidae). Papéis Avulsos de Zoologia 51(7): 101138.

Carvalho MR, UL Gomes \& JD McEachran. 2006. First report and description of a legskate, genus Cruriraja, from the southwestern Atlantic Ocean (Chondrichthyes: Rajidae). Cybium 30(4): 343-354.

Garman S. 1888. On the lateral-line canal system of the Selachia and Holocephala. Bulletin of the Museum of Comparative Zoology at Harvard College 17(2): 57-119.

Jordan LK. 2008. Comparative morphology of stingray lateral line canal and electrosensory systems. Journal of Morphology 269: 1325-1339.

Jordan LK, AM Kajiura \& MS Gordon. 2009. Functional consequences of structural differences in stingray sensory systems. Part I: Mechanosensory lateral line canals. Journal of Experimental Biology 212: 3037-3043.

Koen-Alonso M, EA Crespo, NA García, SN Pedraza, PA Mariotti, B Berón \& NJ Mora. 2001. Food habits of Dipturus chilensis (Pisces: Rajidae) off Patagonia, Argentina. Journal of Marine Science 58: 288-297.

Lloris D \& J Rucabado. 1991. Ictiofauna del canal Beagle (Tierra del Fuego), aspectos ecológicos y análisis biogeográfico. Publicación Especial, Instituto Español de Oceanografía 8: 1-182.

McGowan DW \& S M Kajiura. 2009. Electroreception in the euryhaline stingray, Dasyatis sabina. The Journal of Experimental Biology 212: 1544-1552.

Maruska KP. 2001. Morphology of the mechanosensory lateral line system in elasmobranch fishes: ecological and behavioral consideration. Environmental Biology of Fishes 68: 47-75.

Maruska KP \& TC Tricas. 1998. Morphology of the mechanosensory lateral line system in the Atlantic stingray, Dasyatis sabina: The mechanotactile hypothesis. Journal of Morphology 238: 1-22.

Maruska KP \& TC Tricas. 2004. Test of the mechanotactile hypothesis: neuromast morphology and response dynamics of mechanosensory lateral line primary afferents in the stingray. The Journal of Experimental Biology 207: 3463-3476.

Marzullo T, B Wueringer, L Squire Jnr \& SP Collin. 2011. Description of the mechanoreceptive lateral line and electroreceptive ampullary systems in the freshwater whipray, Himantura dalyensis. Marine and Freshwater Research 62: 771-779.

Montgomery JC \& D Bodznick. 1999. Signals and noise in the elasmobranch electrosensory system. The Journal of Experimental Biology 202: 1349-1355.

Raschi W. 1986. A morphological analysis of the ampullae of Lorenzini in selected skates (Pisces, Rajoidei). Journal of Morphology 189: 225-247.

Reyes P \& M Hüne. 2012. Peces del sur de Chile, 500 pp. Ocho Libros Editores, Santiago.

Roberts BL. 1978. Mechanoreceptors and the behavior of elasmobranch fishes with special reference to the acousticlateralis system. In: Hodgson ES \& RF Mathewson (eds). Sensory biology of sharks, skates and rays, pp. 331-390. US Government Printing Office, Washington.

Shibuya A \& S Tanaka. 2012. Distribution of the lateral line canals in Dasyatis matsubarai (Elasmobranchii, Dasyatidae) from Japanese waters. Pan-American Journal of Aquatic Sciences 7(3): 178-181.

Shibuya A, J Zuanon, MLG de Araújo \& S Tanaka. 2010. Morphology of the lateral line canals in Neotropical freshwater stingrays (Chondrichthyes: Potamotrygonidae) from Negro River, Brazilian Amazon. Neotropical Ichthyology 8(4): 867-876.

Tricas TC. 2001. The neuroecology of the elasmobranch electrosensory world: why peripheral morphology shapes behavior. Environmental Biology of Fishes 60: 77-92.

Wueringer BE \& IR Tibbetts. 2008. Comparison of the lateral line and ampullary systems of two species of shovelnose ray. Reviews in Fish Biology and Fisheries 18(1): 47-64.

Wueringer BE, SC Peverell, J Seymour, L Squire Jr., L Kajiura \& SP Collin. 2011. Sensory system in sawfishes. 1. The ampullae of Lorenzini. Brain, Behavior and Evolution 78: 139-149. 